\title{
Mesenchymal Stem Cell therapy for COVID-19- induced ARDS patients. A successful phase1, randomized, control-placebo group, clinical trial
}

Najmeh Kaffash Farkhad

Mashhad University of Medical Sciences

Alireza Sedaghat

Mashhad University of Medical Sciences

Hamidreza Reihani

Mashhad University of Medical Sciences

Amir Adhami Moghadam

Mashhad University of Medical Sciences

Ahmad Bagheri Moghadam

Mashhad University of Medical Sciences

Nayereh Khadem Ghaebi

Mashhad University of Medical Sciences

Mohamad Ali Khodadoust

Mashhad University of Medical Sciences

Rashin Ganjali

Mashhad University of Medical Sciences

Amirreza Tafreshian

Mashhad University of Medical Sciences

Jalil Tavakol Afshari ( $\sim$ Tavakolaj@mums.ac.ir)

Mashhad University of Medical Sciences

\section{Research Article}

Keywords: Mesenchymal Stem Cells, Safety, Efficacy, Clinical trial, Acute respiratory distress syndrome

Posted Date: January 24th, 2022

DOI: https://doi.org/10.21203/rs.3.rs-1240880/v1

License: (1) (1) This work is licensed under a Creative Commons Attribution 4.0 International License.

Read Full License 


\section{Abstract}

\section{Background}

Acute respiratory distress syndrome (ARDS) is the devasting complication of the new COVID-19 pandemic, directly correlated with releasing large amounts of inflammatory cytokines. Due to their immunoregulatory features, Mesenchymal Stem Cells (MSCs) provide a promising approach against this disease. In this regard, this study was designed as a single-center, open-label, phase 1 clinical trial with a control group to examine the safety and efficacy of three injections of Umbilical Cord-derived MSCs (UCMSCs) in mild-moderate COVID-19-induced ARDS patients.

Methods

Twenty confirmed COVID-19 patients with mild to moderate ARDS degree (using the WHO guideline for ARDS classification) entered the study and were randomly divided into two groups: control group (standard care) and intervention group (standard care + UC-MSCs). The patients received three intravenous infusions of UC-MSCs $\left(1 \times 10^{6}\right.$ cells/kg BW per injection) every other day. Respiratory markers, CRP levels and some serum cytokines were assessed four times (days of 0,5,10 and 17) during the 17 days follow-up period.

Results

During the study, there were no serious adverse effects after cell transplantations. Besides, significant improvement in $\mathrm{SPO}_{2} / \mathrm{FIO}_{2}$ ratio and serum CRP levels was observed. On the other hand, a significant decrease $(P<0.05)$ in serum cytokine levels of IL-6, IFN-g, TNF-a, IL-17 A and a significant increase in serum cytokine levels of TGF-B, IL-1B and IL-10 was observed. Also, no significant changes were observed in CT scan images of patients during the study period.

Conclusion

Our obtained results demonstrated that multiple intravenous transplantations of allogenic UC-MSCs in non-severe COVID-19-induced ARDS patients are a safe procedure. In addition, this intervention is a hopeful approach to decline cytokine storm and recover respiratory functions. Indeed, more clinical trials with larger sample sizes are required to confirm these results.

Trial registration

This clinical trial was registered with the Iranian Registry of Clinical Trials (ID: IRCT20160809029275N1 at 2020.05.30).

\section{Introduction}


COVID-19, a viral infectious disease caused by a new coronavirus (SARS-CoV-2), first appeared in December 2019 in China $(1,2)$ and quickly became a global pandemic $(3,4)$. The lower respiratory tract, especially the lungs, are the key targets (5) which are easily accessible by the virus via AngiotensinConverting Enzyme 2 (ACE-2) receptor (6). Through the abundance of ACE2 on different organs like the kidney, nervous system, lungs, liver, and heart, multi-organ failure is a relatively common complexity of these patients when the disease progresses severely (6). Acute Respiratory Distress Syndrome (ARDS), the devasting characteristic complexity of COVID-19 disease with high mortality (7) is caused by different mechanisms, including overactivation of neutrophils, the release of a large number of inflammatory cytokines (cytokine storm), and the renin-angiotensin system dysregulation (8). ARDS is a generalized destructive lung injury characterized by pulmonary edema and endothelial damage that leads to progressive respiratory failure (9). It has been proven that among COVID-19 patients, ARDS is a common and crucial reason for the intensive care unit (ICU) admittion of COVID-19 patients $(10,11)$. In this regard, applying therapeutic methods to reduce the inflammatory status and regenerate the lungs can be a useful treatment strategy for COVID-19-induced ARDS patients. MSCs are considered suitable therapeutic candidates in this field due to their immunomodulatory properties.

MSCs are non-hematopoietic, qualified cells characterized by self-renewal ability and potential of differentiating into multiple cell lines (12). So far, they have effectively been used in several studies for treatment of numerous immunologic based disorders, including systemic lupus erythematosus (SLE) (13), Amyotrophic lateral sclerosis (ALS) (14), and graft-versus-host disease (GVHD) (15). In this regard, many researches have also been registered on the use of these cells for COVID-19 patients (https://clinicaltrials.gov), which only a few of them have been completed and published their results (16, 17). It has been revealed that MSCs are ACE2 negative (7) and can effectively inhibit the over-activated immune system in COVID-19 patients (18). Also, the intravenous (IV) injection of MSCs can rapidly carry a high number of them to the lungs (19) as the main injured organs in ARDS (20). Based on these trials and available information, in this study we aim to evaluate the safety and effectiveness of three doses injection of UC-MSCs in COVID-19-induced ARDS patients.

\section{Materials And Methods}

\subsection{Study protocol and participants eligibility}

This study was a single-center, open-label, phase 1, randomized clinical trial with a placebo control group conducted at Imam Reza Hospital, Mashhad University of Medical Sciences, Mashhad, Iran, from July 2020 to May 2021. It was performed following the Declaration of Helsinki Ethical Principles and approved by the Ethics Committee of the Mashhad University of Medical Sciences, Mashhad, Iran (IR. MUMS. REC.1399.150). Also, this clinical trial was registered with the Iranian Registry of Clinical Trials (ID: IRCT20160809029275N1 at 2020.05.30). All patients or their representatives signed an informed consent form to participate in this clinical trial. 
In this trial, 20 pneumonia COVID-19 patients confirmed by RT-PCR or CT scan image with non-severe ARDS (120 $\left.\leq \mathrm{SPO}_{2} / \mathrm{FIO}_{2} \leq 315\right)$ were considered eligible and randomly divided into two groups, including a standard care group (control group) and standard care plus umbilical cord mesenchymal stem cell infusion group (intervention group). All patients were admitted to the ICU during the study.The inclusion and exclusion criteria are:

\section{Inclusion criteria:}

-Male or female in the age range of 18 to 75 years.

-Signing an informed written consent form.

-Confirmed COVID-19 patients with non-severe ARDS (120 $\left.\leq \mathrm{SPO}_{2} / \mathrm{FIO}_{2} \leq 315\right)$.

-Required supplemental oxygen.

\section{Exclusion criteria:}

-Severe underlying diseases (like auto-immune disease, cancer, heart, liver or kidney dysfunction).

-Pregnancy or breastfeeding.

-Evidence of severe side effects after cell transplantation.

-Concurrent enrolment in other studies.

-Co-infection with other viruses (like HIV, HBS, HBV, influenza virus and etc.)

2.2 UC-MSCs isolation, preparation, characterization, and transplantation

UC-MSCs were obtained from the umbilical cord tissues of healthy mothers with informed consent and prepared for injection. Briefly, the umbilical cords were washed several times with PBS to obtain clean, bloodless tissue. The blood vessels were delicately separated by a longitudinal and transverse incision of the Wharton's jelly. Then it was cut up into $1-2 \mathrm{~mm}$ pieces and treated respectively with $0.2 \%$ Collagenase/Dispase (Boehringer Mannhetm GombH, Germany) for 1hour and then $0.125 \%$ Trypsin-EDTA (Gibco, USA) for 30 minutes at $37^{\circ} \mathrm{C}$ with agitation. After centrifugation (1500 rpm, 5 minutes) and remove the supernatant (3 times), small pieces were seeded in $25 \mathrm{~cm}^{2}$ flasks (SLP, South Corea) and maintained in a culture medium including minimum essential medium-a (alpha-MEM, Biowest/ South America) supplemented with $20 \%$ fetal bovine serum (Biowest/ South America) and $1 \%$ penicillin/streptomycin (Gibco, USA), and then incubated in a humidified incubator at $37^{\circ} \mathrm{C}$ under $5 \% \mathrm{CO} 2$. After observing the several Colony Forming Units (CFU), the tissues were removed from the culture flasks. The fibroblast-like adherent cells were digested and re-plated for expansion to passage 3 (21). Then UCMSCs were characterized based on the International Society for Cellular Therapy (ISCT) Guidelines by flow cytometry analysis (Fig.1) and differentiation ability into osteocytes (Fig.2.A) and adipocytes 
(Fig.2.B) (21). Briefly, osteogenic differentiation of MSCs was assessed with alizarin red S (Kia zist, Iran) staining. In addition, oil red O (Kia zist, Iran) staining was used to examine the capacity of MSCs to differentiate along an adipogenic lineage.

To ensure sterility and lack of bacterial, yeast, fungal, and mycoplasma contamination, endotoxin, Bactec, and PCR tests, were performed on the cell samples before each injection. The cell viability percentage was between 95 and $98 \%$ before each infusion. Finally, after reaching $80 \%$ confluence, the UC-MSCs were detached using $0.125 \%$ trypsin-EDTA (Gibco, USA) and were suspended in $100 \mathrm{ml}$ of normal saline for each infusion. Intravenously three injections $(1 \times$ cells $/ \mathrm{kg} \mathrm{BW}$ per injection) every other day (days 1, 3, and 5) was performed for patients. The infusion time was approximately 30-45 min (approximately 50 drops/min). In a placebo-control group, $100 \mathrm{ml}$ of normal saline was injected concurrently. In all stages of the study, patients got standard care based on their particular situations. All the procedures mentioned above were performed in a Grade B cleanroom with GMP requirements.

(Dott line: unstained control, Solid line: a marker of interest ).

\subsection{Assessment of clinical and paraclinical parameters and follow up period}

The main aim of the present study was to investigate the safety and effectiveness of 3 doses of UCMSCs transplantation with an interval of one day in COVID-19-induced ARDS patients. For this purpose, to check safety, patients were closely monitored by a physician for 24 to 48 hours after each injection for possible allergic reactions, hypotension, severe headache and/or severe adverse effects like anaphylactic reactions. If any of these symptoms were observed, the patient was immediately excluded from the study. Additionally, to evaluate the efficacy, patients' respiratory parameters, including the Spo2/Fio2 ratio and patients ' pre and post lung status, were assessed. Changes in the serum CRP levels and level of inflammatory and proinflammatory cytokines during the treatment period were also examined (ELISA, Karmania Pars gene, Iran). All relevant factors were measured four times on 0, 2, 7, and 14 days after the second injection (days $0,5,10$, and 17). However, the results of CT scan images were evaluated in two stages (before the intervention and two weeks after the intervention).

\subsection{Statistical analysis}

Shapiro-Wilk test was applied to test the normality distribution. Generalized Linear Model (GLM) and Repeated Measure ANOVA (RM ANOVA) analysis were used to test the effect of time on normally distributed data. Non-normally distributed continuous variables are reported as the median (interquartile range, IQR). The comparison of the medians between two related groups was made by paired T-test or Wilcoxon signed ranks. Continuous variables are expressed as the mean (for normally distributed data) or median (for non-normal distributed data) \pm SEM (standard error of the mean/median). The data were analyzed using the statistical package IBM SPSS version 23.0, and the significance level was regarded at $\mathrm{P}<0.05$.

\section{Results}




\subsection{Patients}

In this study, 10 patients participated in the control group ( 4 female and 6 male) with a mean $61.33 \pm$ 5.34 age. Also, in the intervention group, 10 patients ( 3 female and 7 male) with a mean age of $62.00 \pm$ 2.42 were considered eligible and entered the study. During the follow-up period and on the 13th day after the onset of the disease, one death was reported in the control group, while in the intervention group, two patients died on the 6 th and 17th days, respectively. However, examinations by specialist physicians showed no association between death and MSCs injections.

\subsection{Oxygenation}

All participants in this study were in the none-severe group in terms of ARDS index at the onset of the study based on their symptoms and the first ratio of $120 \leq \mathrm{SPO}_{2} / \mathrm{FIO}_{2} \leq 315$, which was in line with WHO guideline (22).

At the beginning of the study, the SPO2/FIO2 ratio was $204.81 \pm 10.84$ in the control group and $186.82 \pm$ 10.61 in the intervention group, which there was no statistically significant difference between these two groups $(P=0.36)$. This ratio significantly decreased to $151.38 \pm 16.05$ in the control group while increased to $223.83 \pm 17.38$ significantly in the intervention group on the 5 th day. Also, a significant difference was observed between these two groups in this time $(P=0.003)$. Changes in the SPO2/FIO2 ratio in the control group on the 10th day $(169.49 \pm 14.08)$ again was significantly decreased compared to the first day $(P=0.045)$ while was significantly $(P=0.029)$ increased in the intervention group $(248.88 \pm 24.3)$ compared to the first day. Finally, on the 17 th day, a non-significant increase $(P=0.055)$ was observed in the control group (226.24 \pm 9.43$)$ compared to the first day of the study, while in the intervention group (259.28 \pm 24.62), a significant increase $(P=0.018)$ was observed at the same time. Figure 1 shows the mentioned changes.

\subsection{Serum CRP levels}

At the study'-onset, the serum CRP levels in the two groups of control (83.36 \pm 9.4$)$ and intervention $(107.73 \pm 7.2)$ showed a significant difference $(P=0.04)$ which after five days compared to day zero showed a significant increase in the control group $(92.55 \pm 8.66, P=0.008)$ and significant decrease in the intervention group $(70.68 \pm 5.82, P=0.005)$. Also on the tenth day, a significant decrease was observed in both control $(71.04 \pm 6.13, P=0.01)$ and intervention $(45.37 \pm 3.95, P=0.008)$ groups. On the seventeenth day, this rate showed a significant decrease in both control $(62.66 \pm 6.77, \mathrm{P}=0.008)$ and intervention $(19.55 \pm 0.89, P=0.005)$ groups. After the intervention, a significant difference $(P<0.05)$ was observed between the two groups at all times and the decrease in CRP levels was greater in the intervention group than in the control group.

\subsection{Lung imaging}

As expected, the CT scan results of the patients showed insignificant changes in the short time. Since the same result was observed in all patients, here the before- and after-CT image of a patient is shown. 


\subsection{IL-6 levels}

The initial measurement revealed that the serum level of cytokine IL- 6 in the control group was (138.906 \pm $30.68)$, which compared to the intervention group $(121.65 \pm 25.78)$ statistically $(P<0.05)$ was no significant difference at the onset of the study. Treatment with UC-MSCs in the intervention group significantly reduced this level on the fifth $(58.70 \pm 12.18, P=0.008)$, tenth $(38.03 \pm 10.3, P<0.001)$, and seventeenth $(8.78 \pm 1.12, \mathrm{P}<0.0001)$ days respectively. Also, in the control group, a decreasing trend in the level of this cytokine was observed during the study period. This decrease on the fifth day (131.63 \pm 37.56) compared to the first day was not statistically significant but on the tenth $(115.28 \pm 20.13, P$ $=0.035)$ and seventeenth days $(62.78 \pm 14.2, P<0.001)$ it was significant. Also, on the tenth and seventeenth days, a significant difference $(P<0.05)$ was observed between the two intervention and control groups and the rate of reduction of this inflammatory cytokine in the intervention group was significantly higher than the control group.

\subsection{IFN-g levels}

During the study, the level of inflammatory cytokine IFN-g in both control and intervention groups in the patient's serum showed a decreasing trend. This amount was $128.37 \pm 23.11$ in the control group and $122.53 \pm 27.11$ in the intervention group at the study onset, with a statistically non-significant difference. Stem cells treatment resulted in a significant reduction in this cytokine on the 5 th $(86.92 \pm 15.12, \mathrm{P}<$ $0.0001)$, 10th $(83.66 \pm 17.14, \mathrm{P}<0.0001)$ and 17 th days $(57.37 \pm 8.9, \mathrm{P}<0.0001)$ compared to before injection. Also, in the control group on the 5th day, there was a non-significant decrease (120.14 \pm 25.47$)$, while on the 10th $(116.8 \pm 20.12, \mathrm{P}<0.001)$ and 17 th $(102.13 \pm 18.94, \mathrm{P}<0.001)$ days, a significant decrease compared to the onset of study was observed. Besides, on the fifth $(P=0.002), 10$ th $(P=0.002)$ and seventeenth $(P<0.0001)$ days, a significant decrease was observed in the intervention group compared to the control group, respectively, which indicates a greater decrease in this cytokine in the intervention group than the control group.

\subsection{TNF-a levels:}

The initial serum level of cytokine TNF-a in the two groups of control $(38.11 \pm 4.17)$ and intervention $(42.33 \pm 5.9)$ showed a non-significant difference. Simultaneously with the disease progression on the fifth day of the study, this cytokine level in the control group increased significantly (44.30 $\pm 6.21, P=$ $0.001)$, but in the intervention group, it decreased non-significantly $(40.81 \pm 4.8, P=0.7)$ compared to the onset of the study. On the 10th day, a non-significant decrease $(37.52 \pm 3.4, \mathrm{P}=0.54)$ in the control group and a significant decrease in the intervention group (34.05 $\pm 5.1, P<0.0001)$ was observed. After 17 days, a significant decrease was observed in both control $(31.20 \pm 5.6, P<0.0001)$ and intervention groups $(18.88 \pm 3.7, P<0.0001)$. Also, on the 17th day, a significant difference $(P<0.0001)$ was observed between the intervention and control groups.

\subsection{IL-1B levels:}


At the study'-onset, there was no significant difference in cytokine IL-1B levels between the control (27.96 $\pm 5.11)$ and intervention groups $(25.70 \pm 7.15)$. The level of this cytokine on the 5 th day of the study increased significantly in control $(31.37 \pm 6.78, \mathrm{P}<0.001)$ and intervention group $(27.49 \pm 8.34, \mathrm{P}<$ $0.001)$. Also, a significant increase was observed on the 10th day in both control (33.08 \pm 7.14$)$ and intervention groups $(28.07 \pm 6.12)$. In addition, on the 17 th day, a significant increasing trend was observed in both control $(33.53 \pm 8.16)$ and intervention groups $(28.20 \pm 7.89)$. On the other hand, in all three time periods of the 5th, tenth and 17th days, there was a significant difference $(P<0.05)$ between the two control and intervention groups and the decreasing trend was more in the intervention group.

\subsection{IL-17 A levels:}

The initial level of inflammatory cytokine IL-17 A in the two groups of control $(43.52 \pm 6.41)$ and intervention ( $41.42 \pm 5.32$ ) showed no statistically significant difference. On the 5 th day, a non-significant increase was observed in both control $(48.75 \pm 7.64, P=0.17)$ and intervention groups $(43.18 \pm 5.11, P=$ 0.9) compared to the first day of the study. Also, on the 10th day of the disease, the level of this cytokine in the control group showed a non-significant decrease $(46.37 \pm 8.12, \mathrm{P}=0.4)$ while in the intervention group showed a significant decrease $(25.76 \pm 5.18, P<0.001)$. On the 17 th day of the disease, a significant decrease in the amount of this cytokine was observed in both control $(32.08 \pm 4.1, P=0.016)$ and intervention groups $(11.51 \pm 1.3, \mathrm{P}<0.001)$.

\subsection{TGF-B levels:}

TGF-B cytokine levels at the beginning of the study were $(23.06 \pm 5.12)$ in control and $(24.38 \pm 6.15)$ in the intervention group, which did not show a statistically significant difference $(P=0.26)$. On the 5th day of the disease, the cytokine level in the control group significantly decreased $(19.89 \pm 4.76, P=0.02)$ while in the intervention group significantly increased $(27.03 \pm 7.24, P=0.02)$. Also, on the 10 th day compared to the first day of study, its level decreased insignificantly in the control group $(22.70 \pm 6.17, P$ $=0.18)$ and increased significantly in the intervention group $(33.20 \pm 8.12, P=0.003)$. On the 17th day, an increasing trend was observed in the levels of this cytokine which this increase was statistically insignificant in the control group (24.24 \pm 7.34 ) but was significant in the intervention group (42.52 \pm $9.11, P=0.005)$. Also, on the 5 th, tenth and 17 th days, a significant difference was observed between the two intervention and control groups and the amount of this cytokine in the intervention group compared to the control group showed a significant increase $(P<0.05)$.

\subsection{IL-10 levels:}

Cytokine IL-10 at the beginning of the study showed an insignificant difference in both controls $(8.19 \pm$ $1.66)$ and intervention groups $(8.57 \pm 1.53)$. After five days of the disease, its rate significantly decreased in the control group $(6.38 \pm 1.23, P=0.004)$ while significantly increased in the intervention group (32.31 $\pm 6.12, P=0.009$ ). On the 10th day of the disease, a non-significant decrease was observed in the control group $(8.06 \pm 2.41, P=0.48)$ and a significant increase in the level of this cytokine in the intervention group $(42.13 \pm 11.32, \mathrm{P}<0.0001)$. Besides, on the 17 th day of the study, compared to the first day, a significant increase was observed in both control $(14.53 \pm 2.8, \mathrm{P}=0.002)$ and intervention groups (68.06 
$\pm 15.12, P<0.0001)$. A significant increase $(P<0.05)$ in the amount of this cytokine was observed in the intervention group on the 5 th, 10 th and 17 th days compared to the control group.

\section{Discussion}

The present study aimed to assess the safety and potency of 3 doses of UC-MSCs in COVID-19 -induced ARDS patients. All participants were non-severe in terms of ARDS index at the onset of the study, which was calculated based on their first ratio of SPO2/FIO2, and they were admitted to the ICU. The obtained results were promising and showed that MSCs transplantation was safe with no major adverse effect in COVID-19 -induced ARDS patients. The mild headache was the most common side effect during the injections, which was eliminated quickly with complementary therapies. In addition, improvement in respiratory function, which is evident by increasing the SPO2/FIO2 ratio after the first injection (Fig. 1), is efficacy indicator of this method. In the same direction, 7 COVID-19 patients who did not get beneficial results from common standard care were included in a pilot study performed by Leng et al. and received $1 \times 10^{6} \mathrm{MSCs} / \mathrm{kg}$ BW. According to our results, no serious side effects were observed during a 14-day follow-up period after injection and disease symptoms greatly improved (7). Furthermore, in another study by Wilson et al. on 9 ARDS patients, injection of 3 courses of MSCs with different doses (low to high) had no injection-related side effects (23).

However, the mortality rate in the intervention group was $20 \%$ (2 out of 10 cases), which was more than the control group with a $10 \%$ mortality rate (1 out of 10 cases), but no significant relationship was observed between cell injections and patients' death. Differences in patients' initial conditions and discrepancy in response to standard treatments can be reasons for differences in mortality rates in these two groups. On the other hand, the mortality rate of patients in this study was less than the study of Hashemian et al. with $45 \%$ mortality (17) and was relatively close to the study of Sánchez-Guijo et al. with $15 \%$ mortality rate (24). Also, the results of patients' CT scan in this study (Fig. 3) did not show significant changes during two weeks which was expected due to the short time interval.

We also, in the present study, examined the changes of some inflammatory and anti-inflammatory cytokines to investigate the "cytokine storm "in COVID-19 patients. It has been documented that "cytokine storm "phenomenon is directly correlated with high morbidity and mortality rate in COVID-19 patients diagnosed with $\operatorname{ARDS}(25,26)$. Cytokine storm as a hostile inflammatory response, characterized by the discharge of a wide stream of accumulated inflammatory cytokines like Interleukin-6 (IL-6), Interleukin-1B (IL-1B), IL-8, Tumor Necrosis Factor-alpha (TNFa), and Interferon-gamma (IFNg) which could enhance the SARS-COV-2 invasion by sending alarming signals to the immune cells and recall them to the inflamed sites (27). Following this aggressive stimulation, immune cells discharge lots of free radicals, which coincides with the disease progression leading to ARDS and multi-organ failure in COVID-19 patients (28, 29). Besides, it is approved that cytokine storms severity is directly associated with the severity of clinical symptoms of COVID-19 patients (30). 
As our results show, the patients' inflammatory cytokines at the study'-onset were relatively high. MSCs injection significantly reduced the level of most of these cytokines (IL-6 (Fig. 4), IFN-g (Fig. 5), TNF-a (Fig. 6), IL-17 A (Fig. 8), except cytokine IL-1B (Fig. 7)) while increasing the serum level of antiinflammatory cytokines including TGF-B (Fig. 9) and IL-10 (Fig. 10), which these results were largely consistent with similar studies. For example, Shu et al. in their clinical trial, performed on 41 COVID-19 patients which 12 of whom received $2 \times 10^{6}$ UC-MSCs, for one time, found that cells could potentially reduce the serum IL-6 and CRP levels and improve CT scan images over a 28-day follow-up period (31). Similarly, in the present study, a significant decrease in serum CRP levels was observed following cell injection (Fig. 2). In addition, another case report study reported that two times intravenous administration of UC-MSCs with convalescent plasma significantly improved the pulmonary microenvironment and recovered pulmonary damage caused by inflammation via cytokine storm inhibition (32). Also, in agreement with our results, obtained results from Lanzoni et al. study revealed that UC-MSCs intravenous transplantation in two different doses could significantly decrease the inflammatory cytokine (TNF-a) and increase the anti-inflammatory cytokine (IL-10) in COVID-19-induced ARDS patients (16).

Also, in our study, we observed a dramatic elevation in serum cytokine IL- 6 and IL-17 levels in patients before the onset of the study. It has been proven that IL- 6 triggers the activation of TH17 cells in the interaction between T cell-dendritic cells (33). In this regard, there is a hypothesis that elevated levels of IL- 6 following the viruses' entry into the immune system may be one of the reasons for elevated TH17 cells in COVID-19 patients (34). Several clinical trials have reported a significant decrease in serum IL-6 levels after MSCs- transplantation $(31,35)$ consistent with our results.

As mentioned above, so far, various studies have shown the potency of MSCs to decrease cytokine storm in COVID-19- induced ARDS patients $(17,36)$. Although the main underlying mechanism in this process is not yet clear, there are several hypotheses in this regard. For instance, a recent study shows that UC-MSCs and their derivatives can potentially suppress monocyte activation, IL-6 production and subsequently inhibit the cytokine storm and improve the clinical condition of patients (37). In addition, MSCs have strong anti-inflammatory properties through paracrine secretion of various anti-inflammatory cytokines such as IL-10, TGF-B, IL-4, and prostaglandin E2 (PGE2) which can modulate the over-active immune system $(38,39)$. In combat to virus-entry into the body, MSCs also secret many soluble factors like TGF-B, PGE2, Nitric Oxide (NO), and Indoleamine2,3- dioxygenase (IDO), which modulate the immune system via two ways $(40,41)$. Firstly, inhibiting the activation and expansion of T-helper 1 (TH1) and TH17 cells reduces the inflammatory cytokines IFN-g and interleukin-17 (42). Secondly, by suppressing Dendritic cell (DC) activation, inhibiting their formation from monocytes, and reducing IL-12 production, MSCs can suppress DCs as the central Antigen Presenting Cells (APCs) (42). Moreover, the documents show that MSCs by homing in the lung through intravenous administration could potentially boost lung function via avoiding pulmonary fibrosis, deserving lung pneumocytes, and enhancing the pulmonary microenvironment $(4,7,36)$. Another protective mechanism of MSCs on lungs is secreting the keratinocyte growth factor which leads to decline in alveolar edema and endothelial permeability (43) 
Our study had limitations and advantages over similar clinical trials. One of the limitations of our study was the short follow-up period of patients, that since our study began in the first peaks of the pandemic and the peak workload of doctors and hospitals, there was no possibility of longer follow-up of patients. However, shorter follow-up periods have also been reported $(7,36,44)$. Also, the study's small sample size due to the newness of the disease and financial issues is another limitation of this study which we suggest to be done with a larger population in future trials. One of the key advantages of this study was using an umbilical cord source for stem cell injections which is a safe and non-invasive method with more MSCs density compared to extracting cells from adipose tissue or bone marrow. Also, the use of fresh cells compared to cryo-preserved cells and having a control group for better data comparison was other advantages of this study.

\section{Conclusion}

In conclusion, our results indicated that MSC therapy in COVID-19- induced ARDS patients could downregulate the disease progression safely due to improvement in respiratory function and cytokine storm reduction. Our results also showed that increasing the number of injections (more than once) can lead to an increase in patients' recovery. Although our study results are promising, further clinical investigations with larger sample size and more extended follow-up periods are required to confirm these results.

\section{Abbreviations}

ARDS: Acute respiratory distress syndrome ; COVID-19: Coronavirus disease 2019; MSCs: Mesenchymal stem cells; UC-MSCs : Umbilical Cord-derived MSCs; CRP: C Reactive Protein; SPO2: oxygen saturation; FIO2: The fraction of inspired oxygen; TNF-a: Tumor necrosis factor-alpha; CT: Computed tomography; SARS-CoV-2: Severe acute respiratory syndrome coronavirus 2; ACE-2: Angiotensin-Converting Enzyme 2; ICUs: Intensive care units; SLE: systemic lupus erythematosus : ALS :Amyotrophic lateral sclerosis; GVHD: Graft-Versus-Host Disease ; WHO: World Health Organization; ISCT: International Society for Cellular Therapy; GMP: Good manufacturing practice; IFNg: Interferon-gamma; TGF-B: Tumor Growth Factor-B; IDO: Indoleamine2,3- dioxygenase.

\section{Declarations}

Acknowledgments: We would like to thank the Iranian council for the development of stem cell sciences and technologies for their relatively financial support of this work (Contract No. 11/55696) also deeply appreciate all the patients participating in this clinical trial, their families, and the nurses and staff of Imam Reza Hospital for their sincere assistance.

\section{Authors' contributions:}

Prof.J.TA designed and critically managed the project. He also provided final approval for quality control of cells before injections and revised the final draft of the manuscript. Dr AR.S,HR.R,A.AM and A.BM were 
responsible for selecting patients, performing injections and treating patients, and all related hospital matters. Prof. N.KH GH was in charge of coordinating and approving the umbilical cords. Dr N.KF, along with Prof J.TA, was in charge of the MSCs extraction, culture and preparation. She was also responsible for receiving patients' informed consent, patients' follow-up, doing laboratory test, data ghadering and analysis and preparing the first draft of manuscript.MA.Kh,R.G and AR.T all participated in laboratory tests. All authors read and approved the final manuscript.

Funding: This study was financially supported by Mashhad University of Medical Sciences, Mashhad,Iran.

Availability of data and materials: All data generated or analyzed during this study are included in this published article.

Ethics approval and consent to participate: This study was performed following the Declaration of Helsinki Ethical Principles and approved by the Ethics Committee of the Mashhad University of Medical Sciences, Mashhad, Iran (IR. MUMS. REC.1399.150). Also, this clinical trial was registered with the Iranian Registry of Clinical Trials (ID: IRCT20160809029275N1 at 2020.05.30). All patients or their representatives signed an informed consent form to participate in this clinical trial.

Consent for publication: Not applicable.

Conflict of Interest: The authors declare no conflict of interest.

\section{References}

1. Li Q, Guan X, Wu P, Wang X, Zhou L, Tong Y, et al. Early transmission dynamics in Wuhan, China, of novel coronavirus-infected pneumonia. New England journal of medicine. 2020.

2. Xu X-W, Wu X-X, Jiang X-G, Xu K-J, Ying L-J, Ma C-L, et al. Clinical findings in a group of patients infected with the 2019 novel coronavirus (SARS-Cov-2) outside of Wuhan, China: retrospective case series. bmj. 2020;368.

3. Holshue ML, DeBolt C, Lindquist S, Lofy KH, Wiesman J, Bruce H, et al. First case of 2019 novel coronavirus in the United States. New England Journal of Medicine. 2020.

4. Farkhad NK, Reihani H, Moghadam AA, Moghadam AB, Tavakol-Afshari J. Are Mesenchymal Stem Cells able to manage Cytokine Storm in COVID-19 patients? A review of recent studies. Regenerative therapy. 2021.18:152-160.

5. Fang Y, Liu H, Huang H, Li H, Saqi A, Qiang L, et al. Distinct stem/progenitor cells proliferate to regenerate the trachea, intrapulmonary airways and alveoli in COVID-19 patients. Cell research. 2020;30(8):705-7. 
6. Zhou P, Yang X-L, Wang X-G, Hu B, Zhang L, Zhang W, et al. A pneumonia outbreak associated with a new coronavirus of probable bat origin. nature. 2020;579(7798):270-3.

7. Leng Z, Zhu R, Hou W, Feng Y, Yang Y, Han Q, et al. Transplantation of ACE2-mesenchymal stem cells improves the outcome of patients with COVID-19 pneumonia. Aging and disease. 2020;11(2):216.

8. Magro G. SARS-CoV-2 and COVID-19: Is interleukin-6 (IL-6) the 'culprit lesion'of ARDS onset? What is there besides Tocilizumab? SGP130Fc. Cytokine: X. 2020;2(2):100029.

9. Kao K-C, Hu H-C, Chang C-H, Hung C-Y, Chiu L-C, Li S-H, et al. Diffuse alveolar damage associated mortality in selected acute respiratory distress syndrome patients with open lung biopsy. Critical Care. 2015;19(1):1-10.

10. Huang C, Wang Y, Li X, Ren L, Zhao J, Hu Y, et al. Clinical features of patients infected with 2019 novel coronavirus in Wuhan, China. The lancet. 2020;395(10223):497-506.

11. Zhou F, Yu T, Du R, Fan G, Liu Y, Liu Z, et al. Clinical course and risk factors for mortality of adult inpatients with COVID-19 in Wuhan, China: a retrospective cohort study. The lancet. 2020;395(10229):1054-62.

12. Farkhad NK, Mahmoudi A, Mahdipour E. How similar are human mesenchymal stem cells derived from different origins? a review of comparative studies. Current stem cell research \& therapy. 2021;16(8):980-93.

13. Manchikanti L, Centeno CJ, Atluri S, Albers SL, Shapiro S, Malanga GA, et al. Bone marrow concentrate (BMC) therapy in musculoskeletal disorders: evidence-based policy position statement of American Society of Interventional Pain Physicians (ASIPP). Pain Physician. 2020:E85-E131.

14. Tavakol-Afshari J, Boroumand AR, Farkhad NK, Moghadam AA, Sahab-Negah S, Gorji A. Safety and efficacy of bone marrow derived-mesenchymal stem cells transplantation in patients with amyotrophic lateral sclerosis. Regenerative Therapy. 2021;18:268-74.

15. Wu Y, Cao Y, Li X, Xu L, Wang Z, Liu P, et al. Cotransplantation of haploidentical hematopoietic and umbilical cord mesenchymal stem cells for severe aplastic anemia: successful engraftment and mild GVHD. Stem cell research. 2014;12(1):132-8.

16. Lanzoni G, Linetsky E, Correa D, Alvarez R, Marttos A, Hirani K, et al. Umbilical cord-derived mesenchymal stem cells for COVID-19 patients with acute respiratory distress syndrome (ARDS). CellR4repair, replacement, regeneration, \& reprogramming. 2020;8.

17. Hashemian S-MR, Aliannejad R, Zarrabi M, Soleimani M, Vosough M, Hosseini S-E, et al. Mesenchymal stem cells derived from perinatal tissues for treatment of critically ill COVID-19-induced ARDS patients: a case series. Stem cell research \& therapy. 2021;12(1):1-12. 
18. Orleans L, is Vice H, Manchikanti L. Expanded umbilical cord mesenchymal stem cells (UC-MSCs) as a therapeutic strategy in managing critically ill COVID-19 patients: the case for compassionate use. Pain physician. 2020;23:E71-E83.

19. Wysoczynki M, Khan A, Bolli R. New paradigms in cell therapy: repeated dosing, intravenous delivery, immunomodulatory actions, and new cell types. Circulation research. 2018;123(2):138-58.

20. Qin $\mathrm{H}$, Zhao A. Mesenchymal stem cell therapy for acute respiratory distress syndrome: from basic to clinics. Protein \& cell. 2020;11:707-22.

21. Seshareddy K, Troyer D, Weiss ML. Method to isolate mesenchymal-like cells from Wharton's Jelly of umbilical cord. Methods in cell biology. 2008;86:101-19.

22. Organization WH. Clinical management of severe acute respiratory infection ( SARI) when COVID19 disease is suspected: interim guidance, 13 March 2020. World Health Organization; 2020.

23. Wilson JG, Liu KD, Zhuo H, Caballero L, McMillan M, Fang X, et al. Mesenchymal stem (stromal) cells for treatment of ARDS: a phase 1 clinical trial. The Lancet Respiratory Medicine. 2015;3(1):24-32.

24. Sánchez-Guijo F, García-Arranz M, López-Parra M, Monedero P, Mata-Martínez C, Santos A, et al. Adipose-derived mesenchymal stromal cells for the treatment of patients with severe SARS-CoV-2 pneumonia requiring mechanical ventilation. A proof of concept study. EClinicalMedicine. 2020;25:100454.

25. Ruan Q, Yang K, Wang W, Jiang L, Song J. Clinical predictors of mortality due to COVID-19 based on an analysis of data of 150 patients from Wuhan, China. Intensive care medicine. 2020;46(5):846-8.

26. Miao Y, Fan L, Li J-Y. Potential treatments for COVID-19 related cytokine storm-beyond corticosteroids. Frontiers in Immunology. 2020;11:1445.

27. Tisoncik JR, Korth MJ, Simmons CP, Farrar J, Martin TR, Katze MG. Into the eye of the cytokine storm. Microbiology and Molecular Biology Reviews. 2012;76(1):16-32.

28. Sinha P, Matthay MA, Calfee CS. Is a "cytokine storm" relevant to COVID-19? JAMA internal medicine. 2020;180(9):1152-4.

29. Ragab D, Salah Eldin H, Taeimah M, Khattab R, Salem R. The COVID-19 cytokine storm; what we know so far. Frontiers in immunology. 2020;11:1446.

30. Liu J, Li S, Liu J, Liang B, Wang X, Wang H, et al. Longitudinal characteristics of lymphocyte responses and cytokine profiles in the peripheral blood of SARS-CoV-2 infected patients. EBioMedicine. 2020;55:102763. 
31. Shu L, Niu C, Li R, Huang T, Wang Y, Huang M, et al. Treatment of severe COVID-19 with human umbilical cord mesenchymal stem cells. Stem cell research \& therapy. 2020;11(1):1-11.

32. Peng $\mathrm{H}$, Gong $\mathrm{T}$, Huang $\mathrm{X}$, Sun $\mathrm{X}$, Luo $\mathrm{H}$, Wang $\mathrm{W}$, et al. A synergistic role of convalescent plasma and mesenchymal stem cells in the treatment of severely ill COVID-19 patients: a clinical case report. Stem Cell Research \& Therapy. 2020;11(1):1-6.

33. Kimura A, Kishimoto T. IL-6: regulator of Treg/Th17 balance. European journal of immunology. 2010;40(7):1830-5.

34. Chi Z, Zhao W, Jia-Wen L, Hong Z, Gui-Qiang W. The Cytokine Release Syndrome (CRS) of Severe COVID-19 and Interleukin-6 Receptor (IL-6R) Antagonist Tocilizumab man be the Key to Reduce the Mortality. https://www ncbi nlm nih gov/pmc/articles/PMC7118634/pdf/main pdf. 2020.

35. Shi L, Huang H, Lu X, Yan X, Jiang $X$, Xu R, et al. Effect of human umbilical cord-derived mesenchymal stem cells on lung damage in severe COVID-19 patients: a randomized, double-blind, placebo-controlled phase 2 trial. Signal transduction and targeted therapy. 2021;6(1):1-9.

36. Chen X, Shan Y, Wen Y, Sun J, Du H. Mesenchymal stem cell therapy in severe COVID-19: A retrospective study of short-term treatment efficacy and side effects. The Journal of Infection. 2020;81(4):647.

37. Shao M, Xu Q, Wu Z, Chen Y, Shu Y, Cao X, et al. Exosomes derived from human umbilical cord mesenchymal stem cells ameliorate IL-6-induced acute liver injury through miR-455-3p. Stem cell research \& therapy. 2020;11(1):1-13.

38. Weiss ARR, Dahlke MH. Immunomodulation by mesenchymal stem cells (MSCs): mechanisms of action of living, apoptotic, and dead MSCs. Frontiers in immunology. 2019;10:1191.

39. Debuc B, Smadja DM. Is COVID-19 a new hematologic disease? Stem cell reviews and reports. 2021;17(1):4-8.

40. Le Blanc K, Mougiakakos D. Multipotent mesenchymal stromal cells and the innate immune system. Nature Reviews Immunology. 2012;12(5):383-96.

41. Zhang W, Ge W, Li C, You S, Liao L, Han Q, et al. Effects of mesenchymal stem cells on differentiation, maturation, and function of human monocyte-derived dendritic cells. Stem cells and development. 2004;13(3):263-71.

42. Jiang $X-X$, Zhang $Y$, Liu B, Zhang $S-X$, Wu $Y$, Yu X-D, et al. Human mesenchymal stem cells inhibit differentiation and function of monocyte-derived dendritic cells. Blood. 2005;105(10):4120-6.

43. Monguió-Tortajada M, Bayes-Genis A, Rosell A, Roura S. Are mesenchymal stem cells and derived extracellular vesicles valuable to halt the COVID-19 inflammatory cascade? Current evidence and future 
perspectives. Thorax. 2021;76(2):196-200.

44. Tao J, Nie Y, Wu H, Cheng L, Qiu Y, Fu J, et al. Umbilical cord blood-derived mesenchymal stem cells in treating a critically ill COVID-19 patient. Journal of Infection in Developing Countries. 2020;14(10).

\section{Figures}
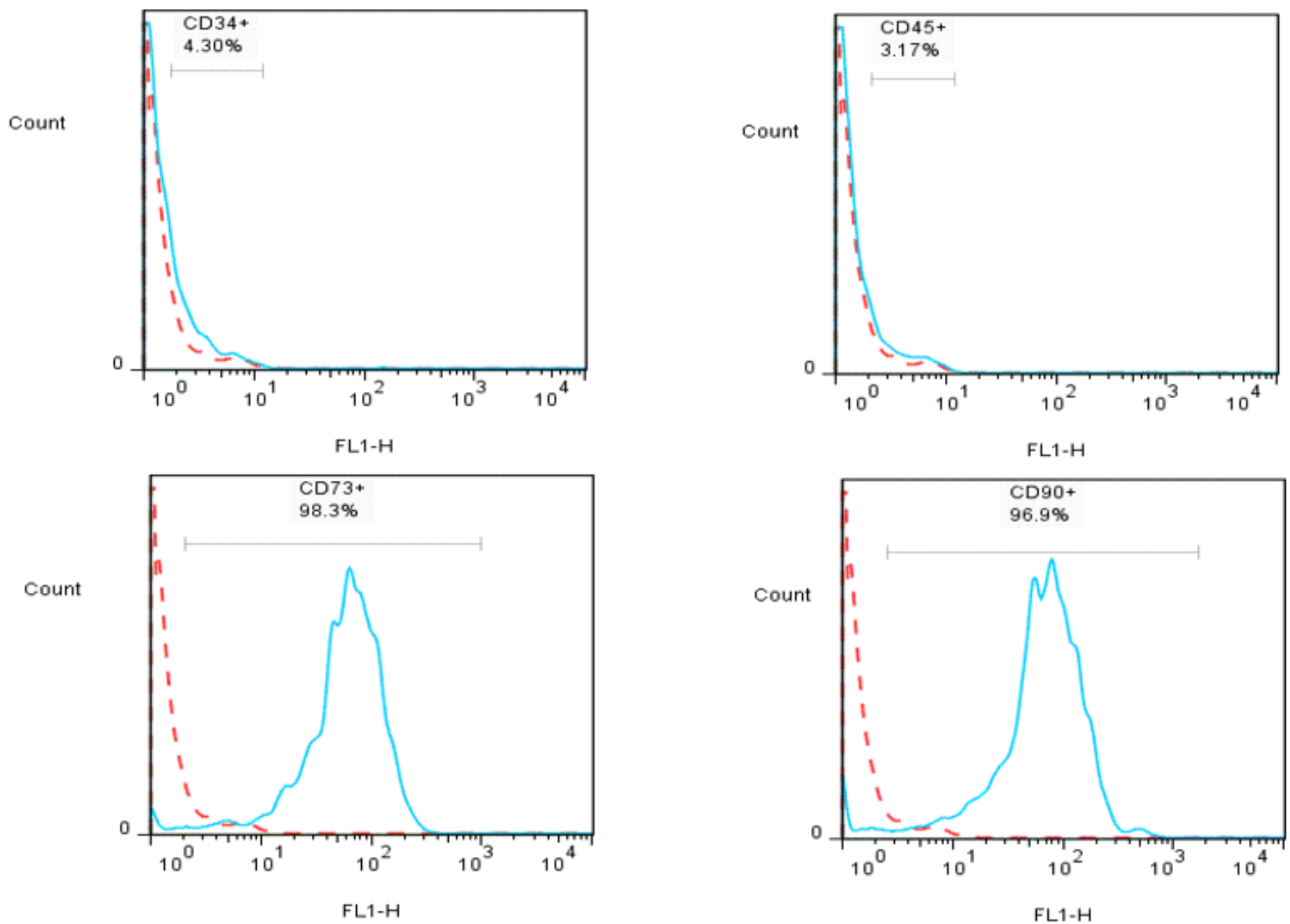

Figure 1

Immunophenotypic characterization of human Umbilical cord-derived Mesenchymal Stem cells by flow cytometry for the expression of mesenchymal ( CD73, CD90) and hematopoietic (CD34, CD 45 ) stem cells markers.

(Dott line: unstained control, Solid line: a marker of interest ). 
A \& $B$ :

A: Osteogenic differentiation and B: Adipogenic differentiation of hUC-MSCs

(Magnification $\times 400$ from Inverted phase microscope)

hUC-MSCs: human Umbilical cord-derived Mesenchymal Stem Cells

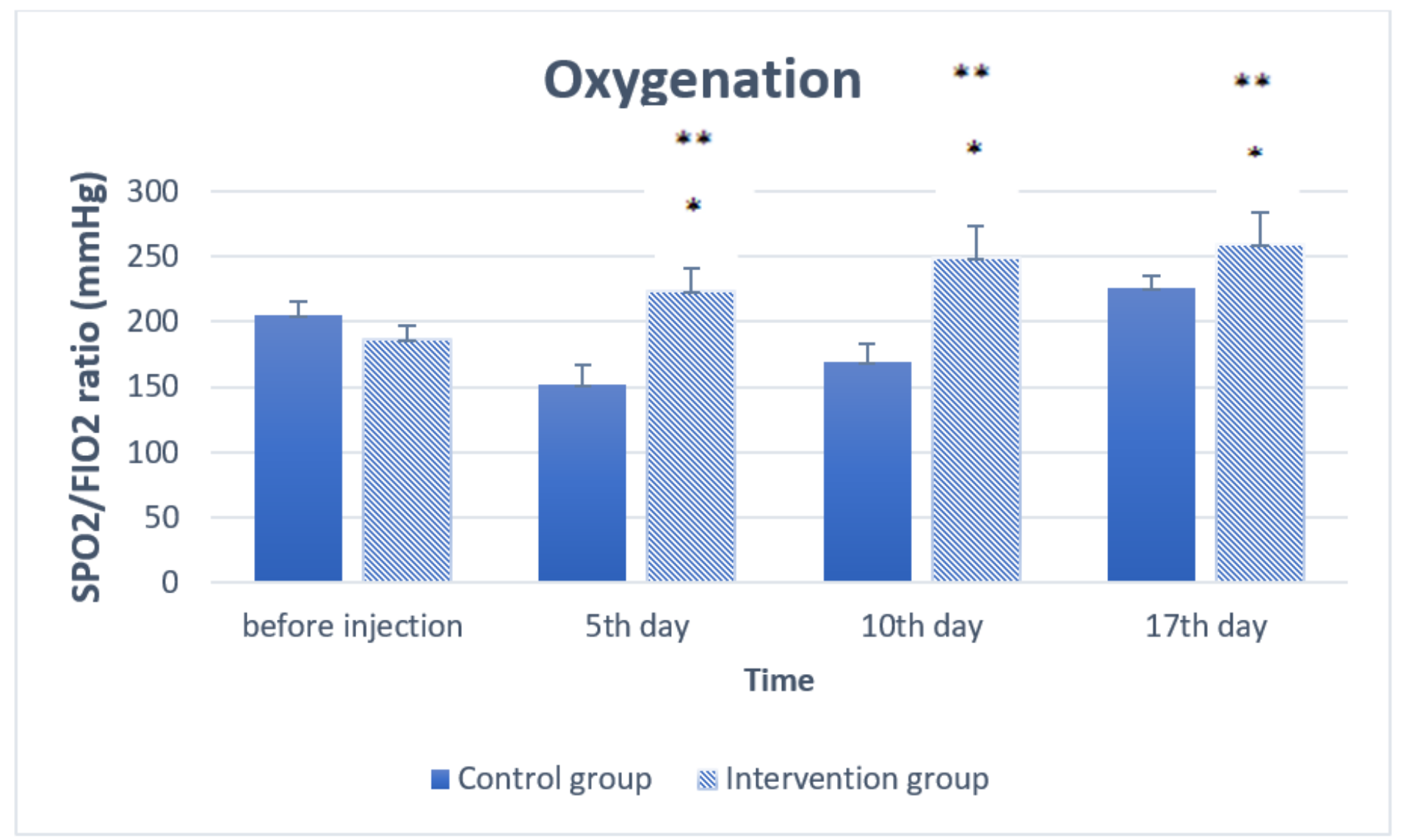

Figure 3

Oxygenation changes (SPO2/FIO2 ratio) in the control and intervention groups during the study. compared to "Control group ": *

compared to "before injection ": **

Figure 5

Shematic lung CT-scan image of a patient. 


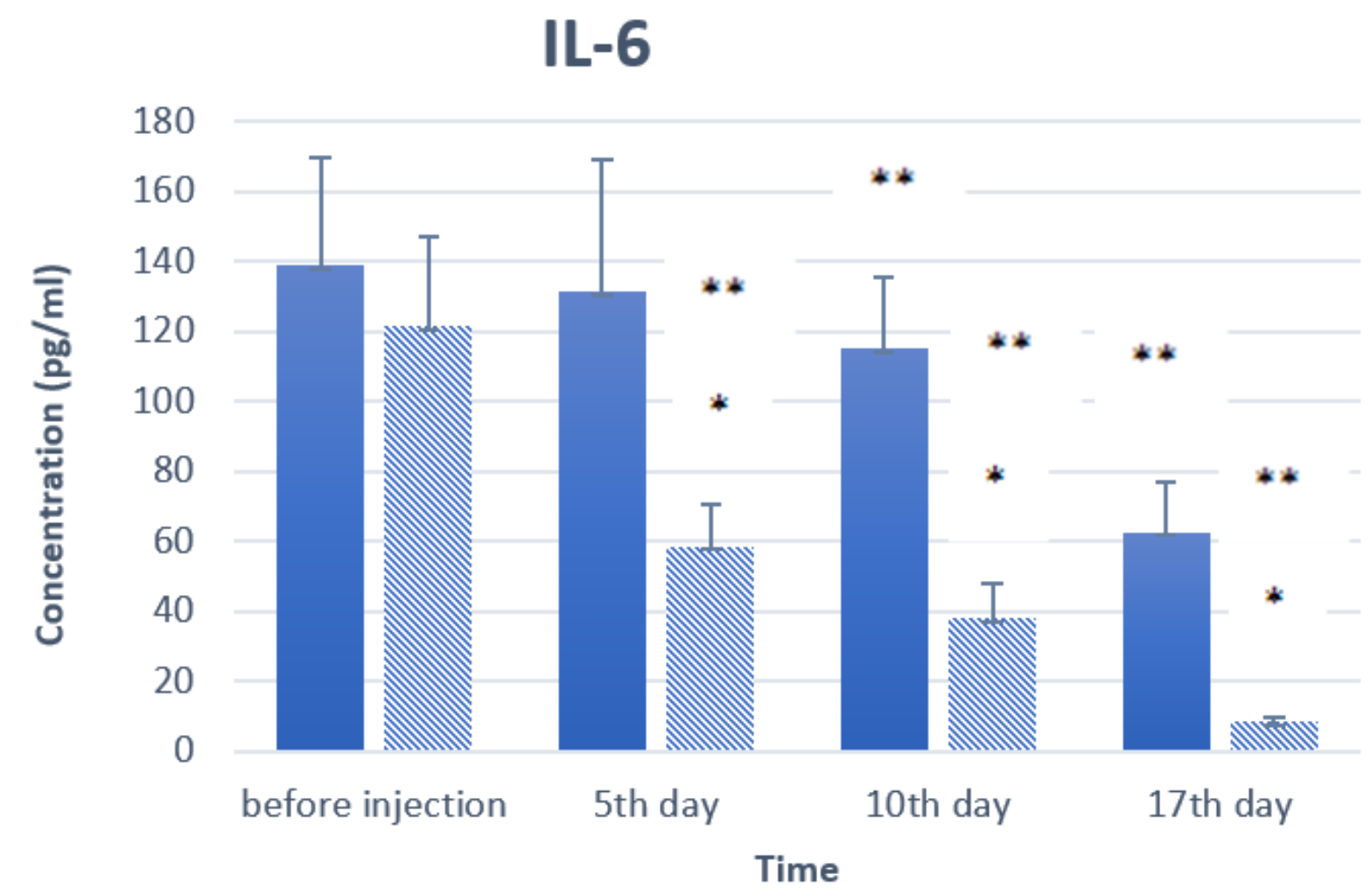

Control group $\mathbb{N}$ Intervention group

Figure 6

Serum levels of TNF-a in the control and intervention groups during the study.

compared to "Control group ": *

compared to "before injection ": ** 


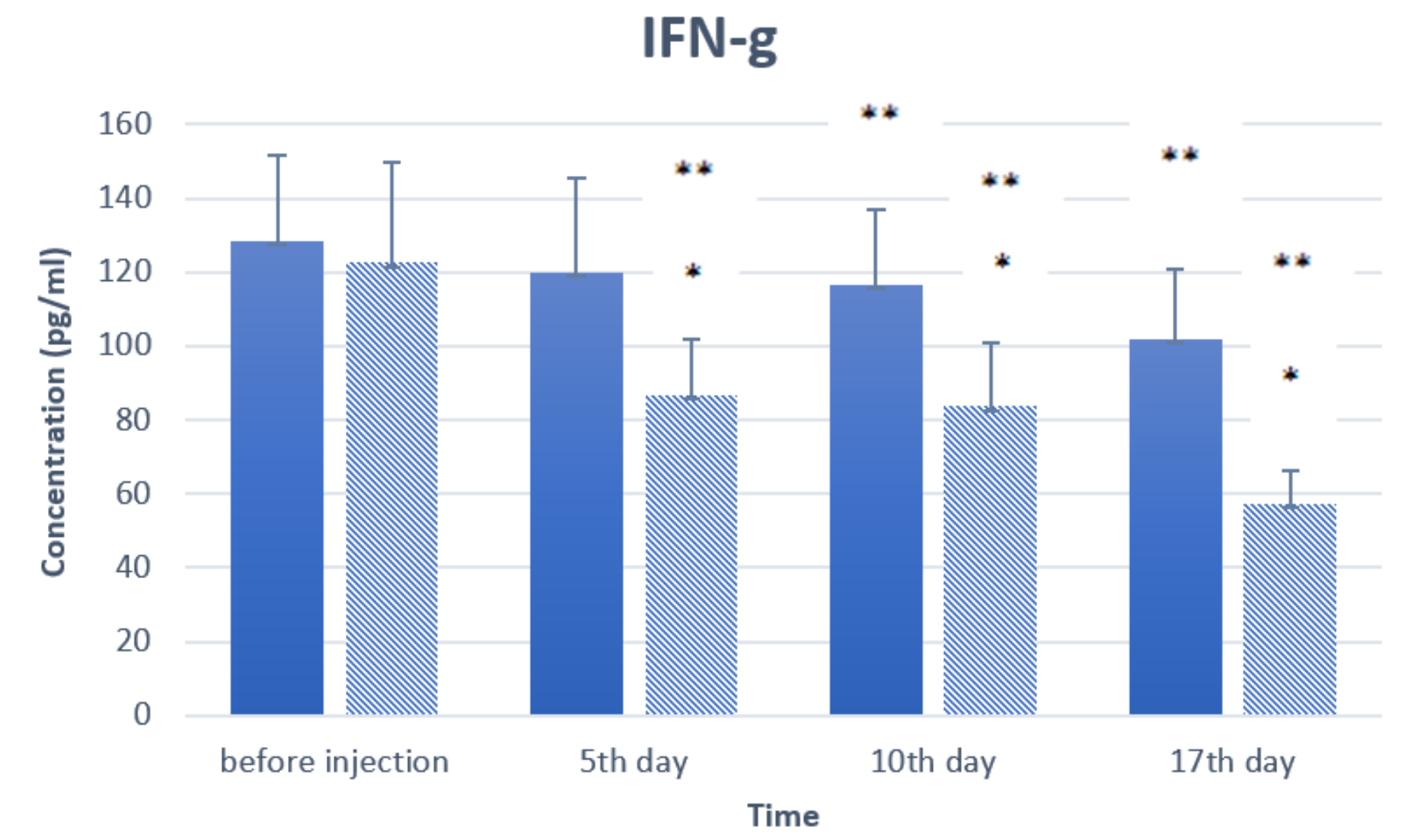

Control group $\mathbb{N}$ Intervention group

Figure 7

Serum levels of IFN-g in the control and intervention groups during the study.

compared to "Control group ": *

compared to "before injection ": ** 


\section{TNF-a}

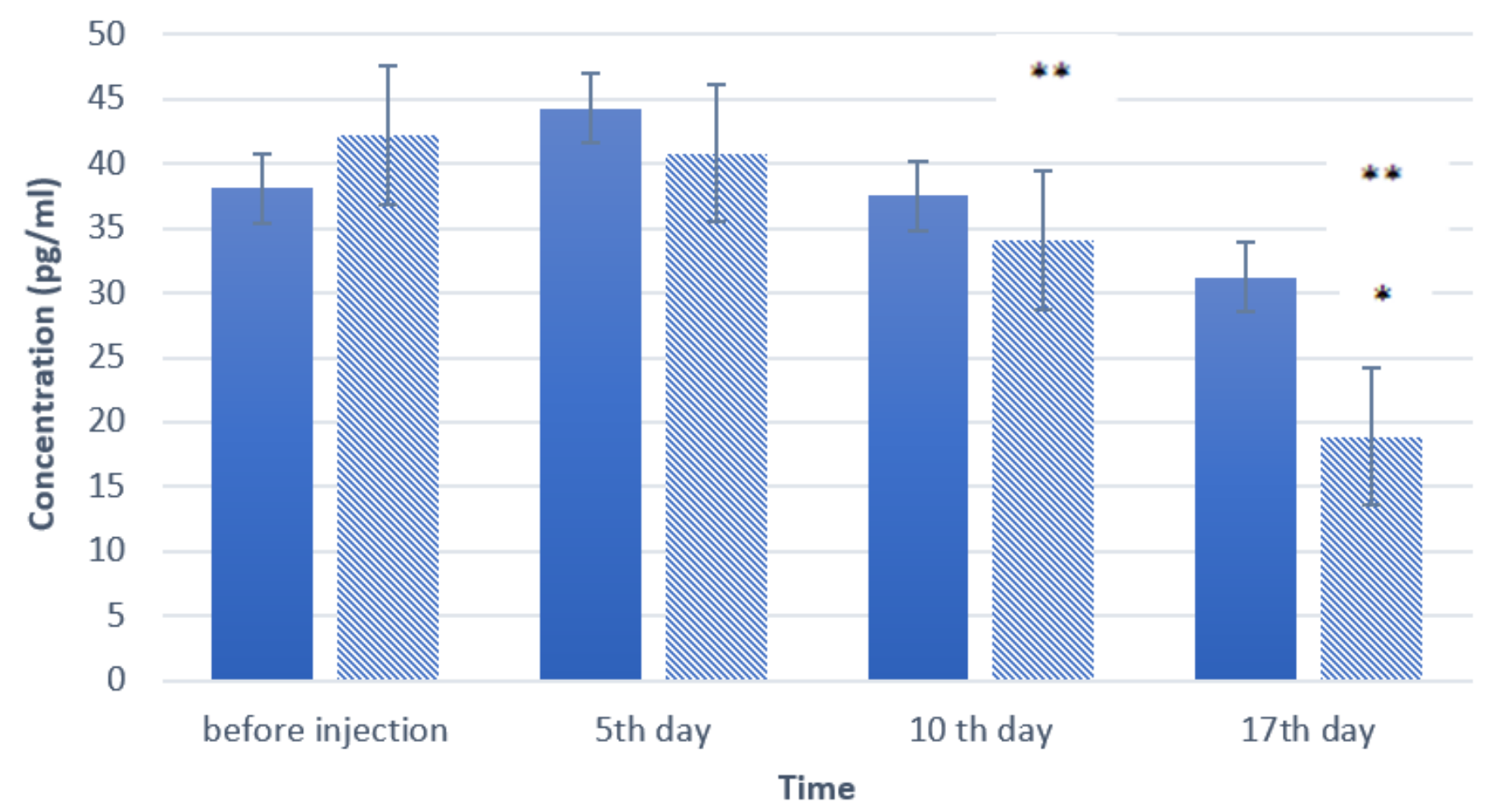

- Control group $\mathbb{N}$ Intervention group

Figure 8

Serum levels of TNF-a in the control and intervention groups during the study.

compared to "Control group ": *

compared to "before injection ": ** 


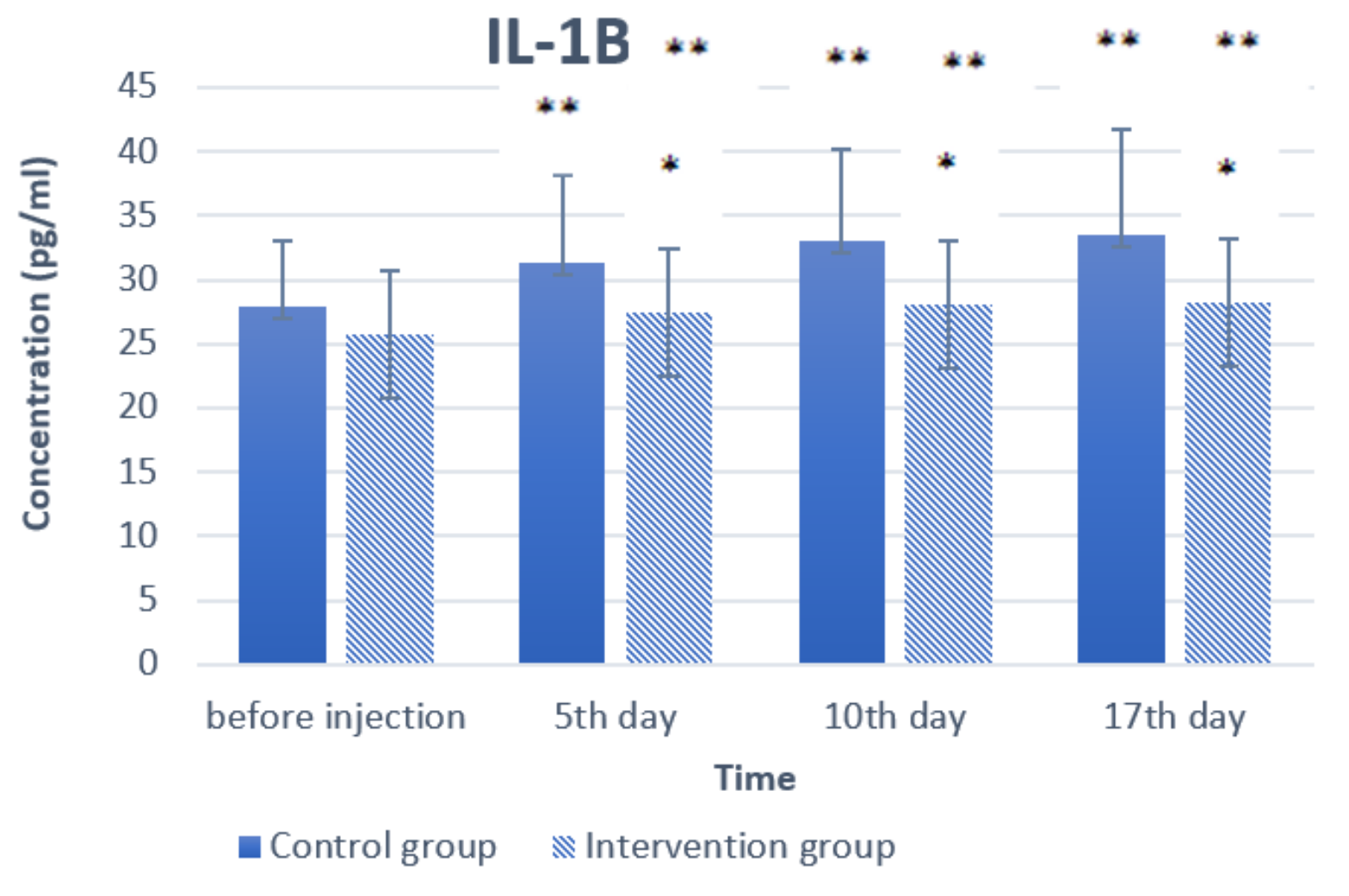

Figure 9

Serum levels of IL-1B in the control and intervention groups during the study.

compared to "Control group ": *

compared to "before injection ": ** 


\section{IL-17A}

70

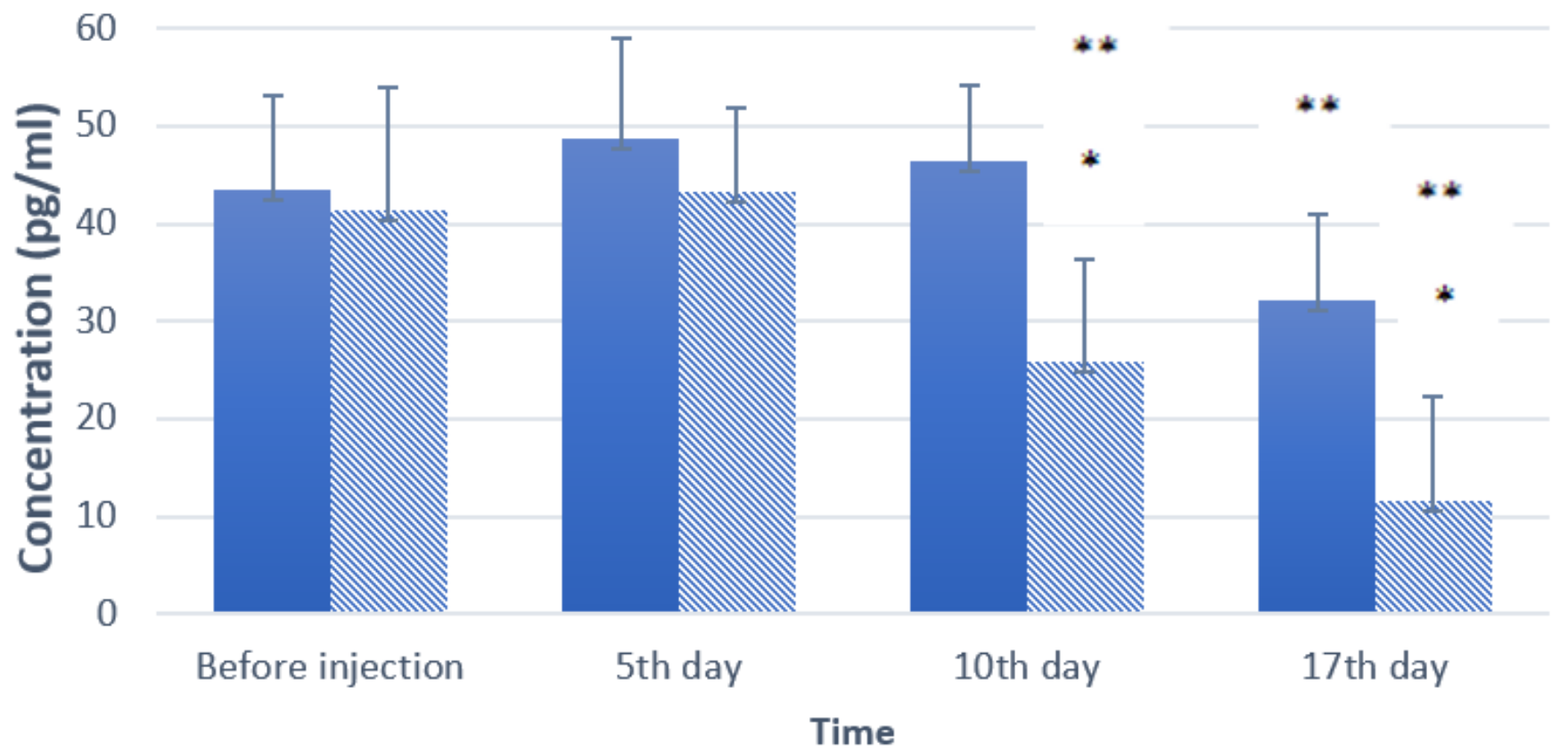

- Control group $\mathbb{N}$ Intervention group

\section{Figure 10}

Serum levels of IL-17 A in the control and intervention groups during the study.

compared to "Control group ": *

compared to "before injection ": ** 


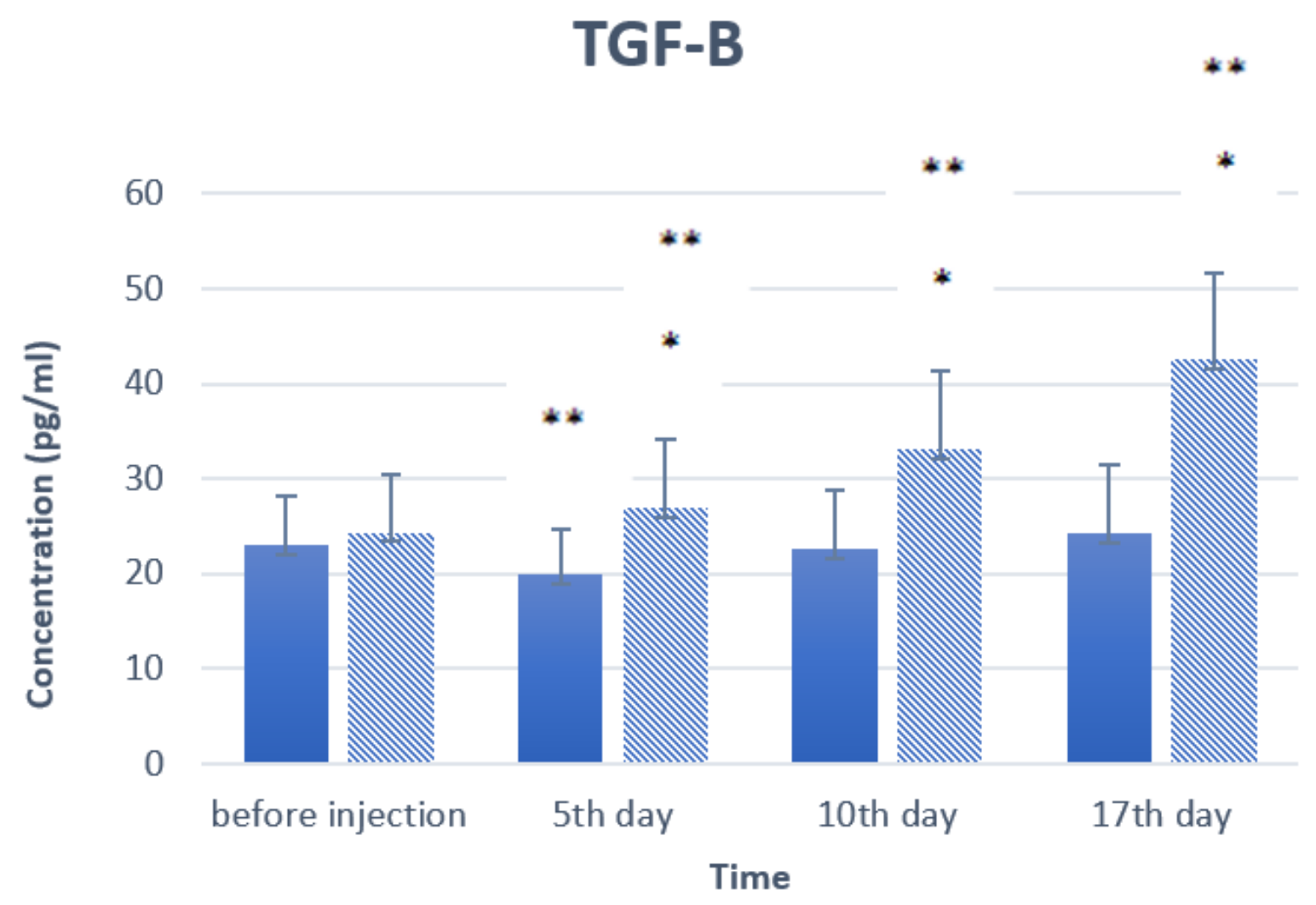

Control group $\mathbb{N}$ Intervention group

Figure 11

Serum levels of TGF-B in the control and intervention groups during the study.

compared to "Control group ": *

compared to "before injection ": ** 


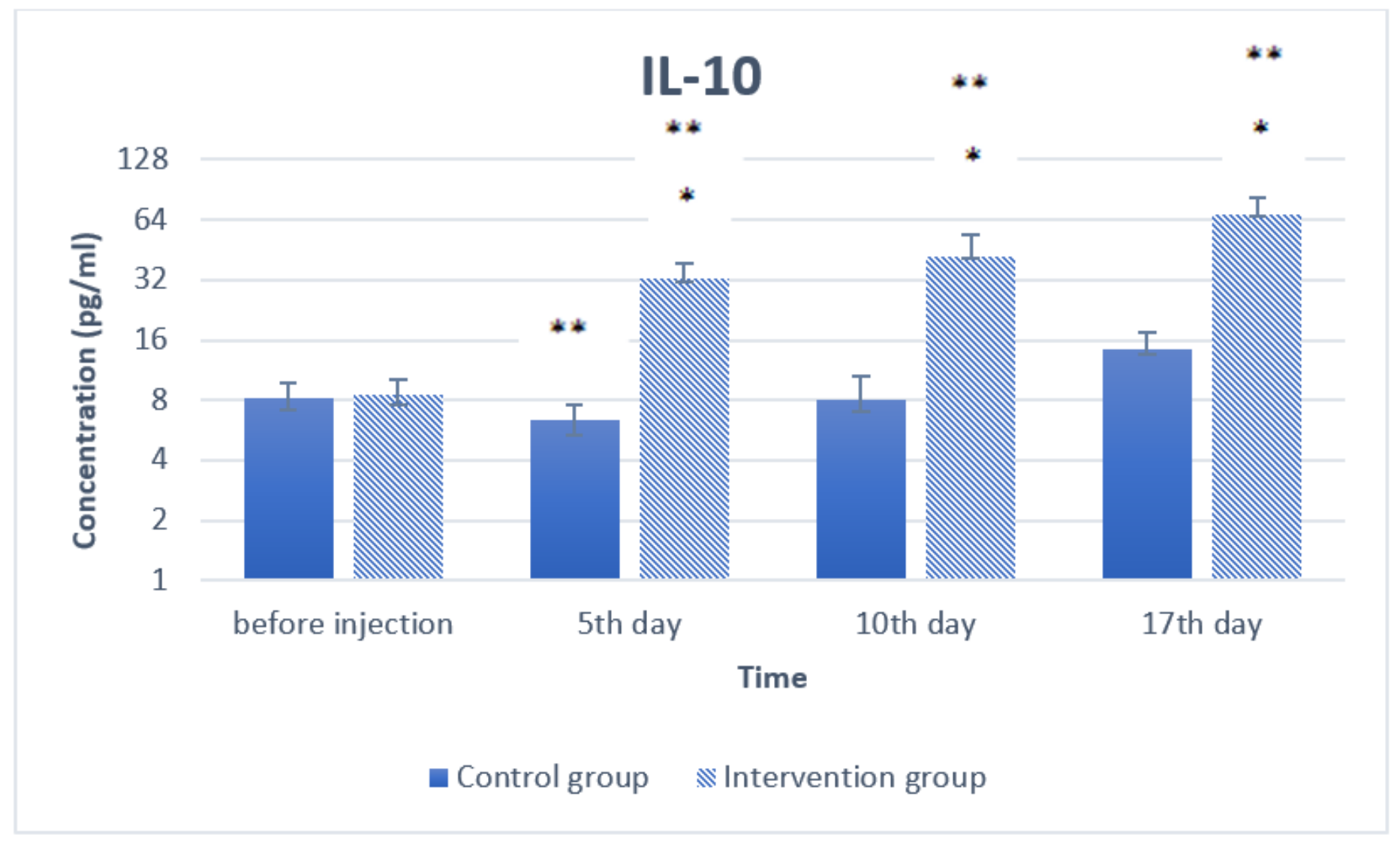

Figure 12

Serum levels of IL-10 in the control and intervention groups during the study. compared to "Control group ": * compared to "before injection ": ** 